\title{
A Novel Dual Stack Sleep Technique for Reactivation Noise suppression in MTCMOS circuits
}

\author{
Tarunnum Sultana ${ }^{1}$, S. Jagadeesh ${ }^{2}$, M.Naveen Kumar $^{3}$ \\ 1. Asst. Prof, Department of ECE, SSJ Engineering college, V. N. Pally, JNTUH - Hyderabad, A.P, \\ 2. Assoc. Prof \& HOD of ECE, SSJ Engineering college, V.N. Pally, JNTUH - Hyderabad, A.P, 500075, \\ 3. M. Tech Student, Department of ECE, SSJ Engineering college, V.N. Pally, JNTUH-Hyderabad, A.P,
}

\begin{abstract}
The development of digital integrated circuits is challenged by higher power consumption as low power circuits are most popular now a days as the scaling increase the leakage power in the circuit also increases rapidly hence various high speed ordered multi-threshold voltage CMOS (MTCMOS) circuit techniques are conferred and evaluated during this paper. Novel dual stack technique is proposed that reduces not only the leakage power but also dynamic power. In this paper, a number of critical considerations in the sleep transistor design and implementation includes header or footer switch selection, Reactivation noise suppression made throughout the sleep to active mode transitions is a vital challenge in MTCMOS circuits.

Keywords: MTCMOS, DUAL STACK, SLEEP TRANSISTOR, DYNAMIC POWER, NOISE SUPPRESSION LEAKAGE POWER.
\end{abstract}

\section{Introduction}

The Scaling of Process Technologies to nanometer regime has resulted in a rapid increase in leakage power dissipation. Hence, it has become extremely important to develop design techniques to reduce static power dissipation during periods of inactivity. The power reduction must be achieved without trading off performance which make NMOS and PMOS transistor harder to reduce leakage during normal (runtime) operation. On the other hand, there are several techniques for reducing leakage power in sleep or standby mode. Power gating in one such well known technique where a sleep transistor is added between actual ground rail and circuit ground. This device is turned off in the sleep mode to cut-off the leakage path. It has been shown that this technique provides a substantial reduction in leakage at a minimal impact on performance.

Power gating technique uses high Vt PMOS sleep transistors which cut off VDD from a circuit block when the block is not switching. The low Vt NMOS sleep transistor sizing is an important design parameter. This technique, also known as MTCMOS [1], or Multi-Threshold CMOS reduces stand-by or leakage power. MTCMOS circuit technique is widely adopted for enhanced energy efficiency in high-performance integrated circuits [1]-[9]. The MTCMOS technique suppresses the leakage currents by disconnecting the idle low threshold voltage (low- $\left.\left|\mathrm{V}_{\text {th }}\right|\right)$ logic gates from the power supply and/or the ground line via cut-off high threshold voltage (high- $\left|\mathrm{V}_{\text {th }}\right|$ ) sleep transistors as shown in Fig 1.

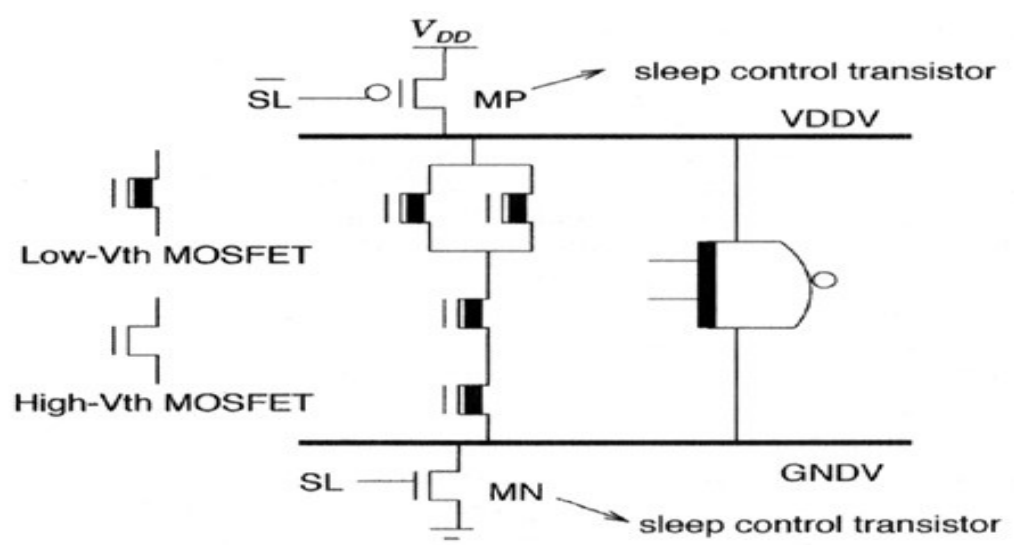

Fig1: MTCMOS technique applied to a logic circuit.

\section{Sleep Transistor Method}

In order to reduce leakage power dissipation we are implementing sleep method in our circuit. In sleep transistor [2][3] method we are connection LOW Vt NMOS transistor between the circuit and the ground 
network. Whenever circuit is in the static position then sleep transistor input becomes zero. This makes ground disconnect from the original circuit thereby reduces the leakage power and shown in Fig 2

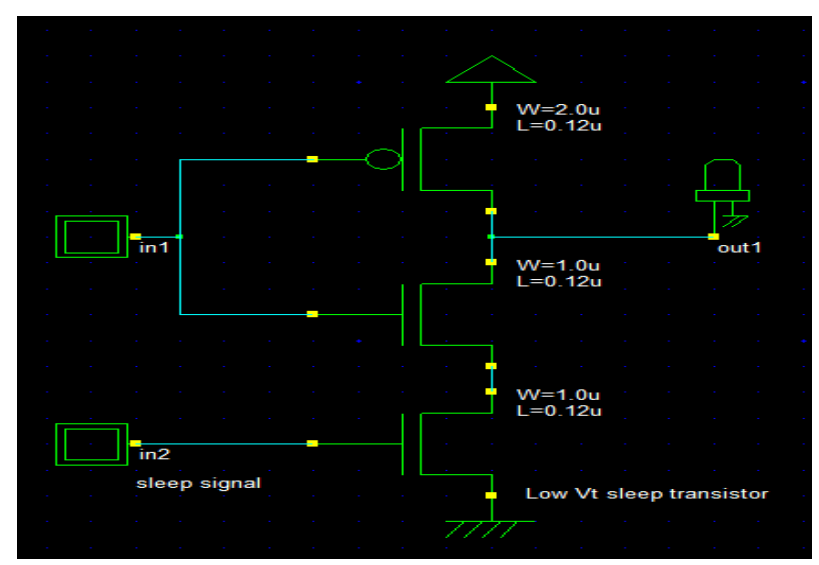

Fig 2: Sleep transistor circuit

At larger circuit during the sleep to active mode of sleep transistor at that time leakage power will dissipates to ground which increase the ground bounce noise effect. To reduce this effect additional modulation circuits are added to sleep signal are illustrated below.

\section{Single Phase Sleep Signal Slew rate modulation}

The effect of input signal instantly rising from 0 to $V_{\mathrm{dd}}$ level will increase the ground bounce noise effect on the circuit. So, the rise time delay of the sleep signal increased the time taken by the sleep transistor to reach saturation region which makes the decrease of ground bounce noise. When the sleep signal [4] gradually rises from $0 \mathrm{~V}$ to $\mathrm{V}_{\mathrm{DD}}$ the voltage level of sleep signal is lower than the threshold voltage ( $\mathrm{V}_{\text {th }}$ sleep) of sleep transistor, the sleep transistor operates in weak inversion region. The virtual ground line (VGND) is discharged very slowly by the small leakage current produced by the high- $\left|\mathrm{V}_{\text {th }}\right|$ sleep transistor the noise induced on the ground distribution network is negligible for $\mathrm{V}_{\mathrm{gs}}<\mathrm{V}_{\text {th }}$ sleep

After the sleep signal rises above the threshold voltage of sleep transistor, the $\mathrm{V}_{\mathrm{GND}}$ is discharged faster. Noise on the real ground is thereby increased. Subsequently, when the $\mathrm{V}_{\mathrm{GND}}$ is discharged to one threshold voltage (low- $\mathrm{V}_{\text {th nMOS }}$ ) below the voltages of the internal nodes in the low- $\left|\mathrm{V}_{\text {th }}\right|$.circuit block, the parasitic capacitors of internal nodes start discharging as well. Another wave of bouncing noise is thereby produced on the real ground wires.

In SLEEP mode, VGND and all the internal nodes of low- $\left|\mathrm{V}_{\text {th }}\right|$ circuit block are maintained at voltage levels close to $V_{\mathrm{DD}}$. During a reactivation event, the internal nodes of the low- $\left|\mathrm{V}_{\mathrm{th}}\right|$ circuit block transition to the correct logic states stage by stage depending on the primary input vectors [7]. Some internal nodes follow the discharging VGND and transition toward $0 \mathrm{~V}$. Significant amount of switching and short-circuit currents are produced by the awakening low- $\left|\mathrm{V}_{\text {th }}\right|$ logic block.

The above Single phase Modulation uses additional circuitry to the existing sleep transistor which indeed increases the power consumption and also area. So, in this paper we are applying a new technique called dual stack technique applied to a SCCER Flip-flop network.

\section{Dual Stack Sleep Technique}

Another technique for leakage power reduction is the stack approach, which forces a stack effect by breaking down an existing transistor into two half Size transistors [7]. The divided transistor NMOS increase delay significantly and could limit the usefulness of the approach. The sleepy stack approach (Fig. 3) combines the sleep and stack approaches $[2,3]$. The sleepy stack technique divides existing transistor NMOS into two half Size transistors like the stack approach. Then sleep transistors are added in parallel to one of the divided transistors. During sleep mode, sleep transistors are turned off and stacked transistors suppress leakage current while saving state. Each sleep transistor, placed in parallel to the one of the stacked transistors, reduces resistance of the path, so delay is decreased during active mode. However, area penalty in NMOS is a significant matter for this approach. Once every transistor is replaced by three transistors and additional wires are added for S and S', which are sleep Signals. This Technique uses the advantage of using the two extra Pull- up and two extra pull-down NMOS and PMOS transistor which works in sleep mode either in OFF state or in ON state.

As a result of stacking, P6 and N6 have less drain voltage. So, the power dissipation effect is less for them and they cause high barrier for leakage current. While in active mode i.e. $=1$ and $S^{\prime}=0$, both the sleep transistors (N5 and P5) and the parallel transistors (N6, N7 and P6, P7) are on. They work as transmission gate 
and the power connection is again established in uncorrupted way. Further they decrease the dynamic power.

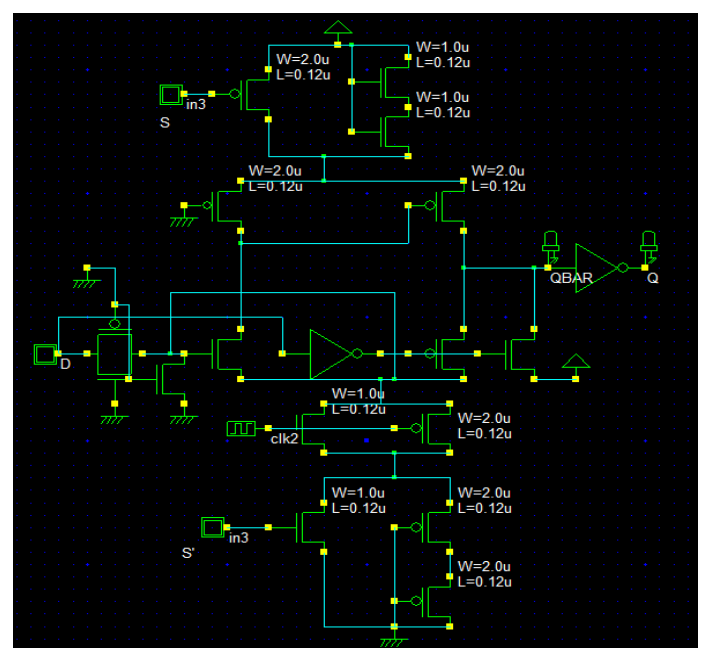

Fig 3: SCCER Flip Flop with Power Gating Circuit

\section{SIMULATION ENVIRONMENT}

The Methodology consists of 3 Modules.

- Logic circuit design, simulation, and Verilog file generation using DSCH.

- Layout design using MICROWIND.

- Simulation.

Here the Conventional technique corresponds to SCEER flip-flop without using Dual Stack approach. In this analysis the circuit is mentioned as Base Circuit. And the Modified technique corresponds to SCCER using Dual Stack Approach and mentioned in the analysis. The proposed design i.e. Novel dual stack approach uses $0.12 \mu \mathrm{m}$ technology and operated with $1.2 \mathrm{~V}$ supply voltage. The simulation parameters have been analyzed with the help of the Microwind tool (version 2) and DSCH (version 2) for the schematic verification.

\section{DSCH (Digital Schematic)}

The DSCH program is a logic editor and simulator. DSCH is used to validate the architecture of the logic circuit before the microelectronics design is started. DSCH provides a user-friendly environment for hierarchical logic design, and fast simulation with delay analysis, which allows the design and validation of complex logic structures

DSCH also features the symbols, models and assembly support for 8051 and 16F84 controllers. The highlights are:-

- Supports Hierarchical logic design.

- Generates a Verilog description of the schematic for layout conversion.

- Immediate access to symbol properties (Delay \& Fan-out)

\section{Microwind}

The Microwind is a tool for designing and simulating circuits at layout level. The tool features full editing facilities (copy, cut, past, duplicate, move), various views (MOS characteristics, 2D cross section, 3D process viewer), and an analog simulator. The Microwind program allows designing and simulating an integrated circuit at physical description level. The package contains a library of common logic and analog ICs to view and simulate. The Microwind includes all the commands for a mask editor as well as original tools never gathered before in a single module (2D and 3D process view, Verilog compiler, tutorial on MOS devices). You can gain access to Circuit Simulation by pressing one single key. 


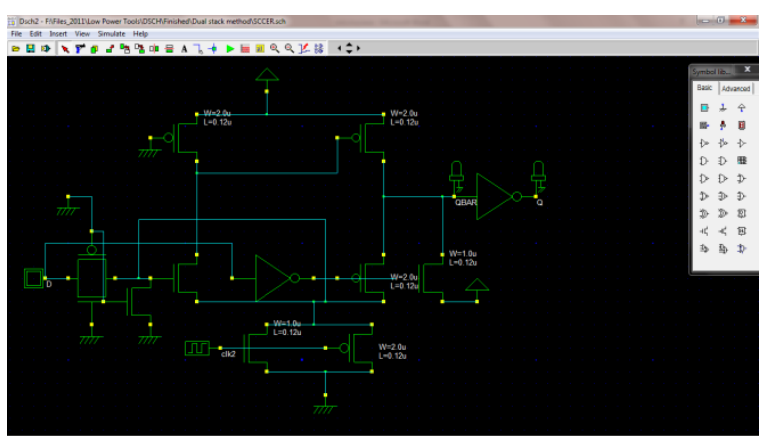

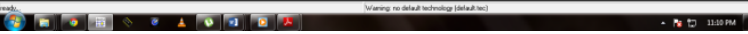

Fig 4: DSCH design of SCCER Filp-Flop

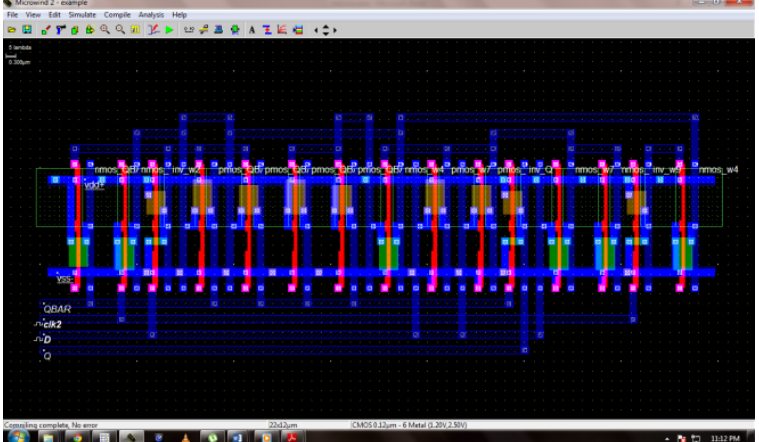

Fig 5: Layout diagram

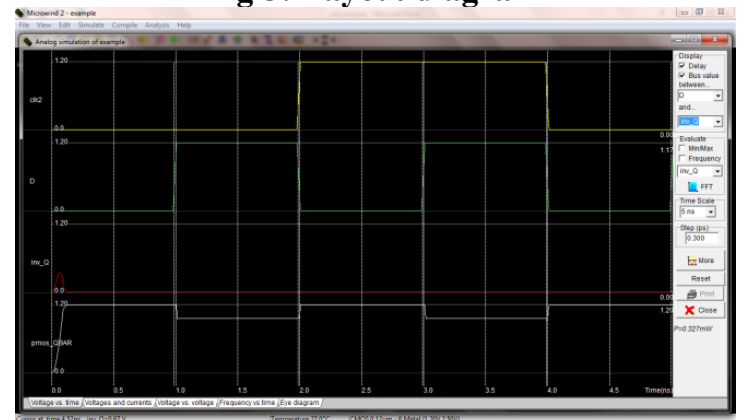

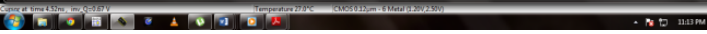

Fig 6: Simulation Wave form in Micro wind

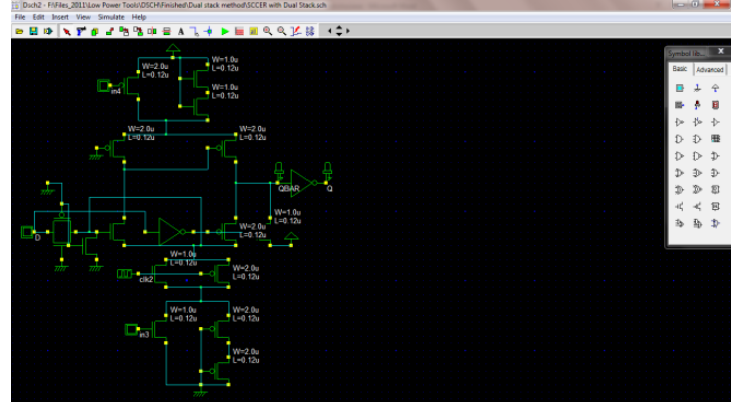

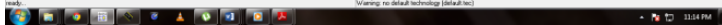

Fig 7: DSCH design of dual stack SCCER Flip-Flop

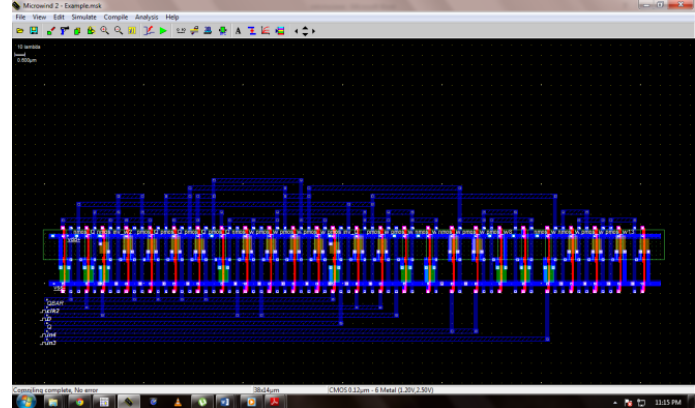

Fig 8: Layout diagram 


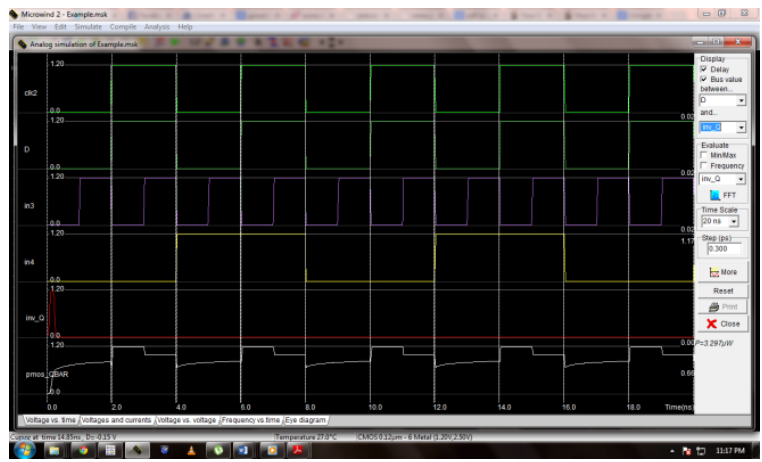

Fig 9: Simulation waveforms in Micro wind

Tabulation of Results

\begin{tabular}{|l|l|l|}
\hline Circuit & Area $\left(\mathbf{u m}^{2}\right)$ & Power Dissipation \\
\hline SCCER & $\mathbf{2 2} * 12$ & $\mathbf{0 . 3 2 7} \mathbf{m W}$ \\
\hline $\begin{array}{l}\text { SCCER With dual } \\
\text { stack }\end{array}$ & $\mathbf{3 8} * 14$ & $\mathbf{3 . 2 9 7} \mathbf{u W}$ \\
\hline
\end{tabular}

\section{Conclusion}

In nanometer scale CMOS technology, sub -threshold leakage power consumption is a great challenge. Although previous approaches are effective in some ways, no perfect solution for reducing leakage power consumption is yet known. Therefore, designers choose techniques based upon technology and design criteria. In this paper, we provide novel circuit structure named "Dual stack" as a new remedy for designers in terms of static power and dynamic powers. Unlike the sleep transistor technique, the dual stack technique retains the original state. The dual stack approach shows the least speed power product among all methods. Therefore, the dual stack technique provides new ways to designers who require ultra-low leakage power consumption

\section{References}

[1] V. Kursun and E. G. Friedman, Multi-Voltage CMOS Circuit Design, John Wiley \& Sons Ltd., 2006, ISBN \# 0-470-01023-1.

[2] S. Mutoh, T. Douseki, Y. Matsuya, T. Aoki, S. Shigematsu, and J. Yamada, "1-V Power Supply High-Speed Digital Circuit Technology," IEEE Journal of Solid-State Circuits, Vol. 30, No. 8, pp. 847-854,August 1995.

[3] J. Kao and A. Chandrakasan, "MTCMOS Sequential Circuits," Proceedings of the European Solid State Circuits Conference, pp. 317 - 320, September 2001.

[4] S. Shigematsu, S. Mutoh, Y. Matsuya, Y. Tanabe, and J. Yamada, "A 1-V High-Speed MTCMOS Circuit Scheme for Power-Down Application Circuits,” IEEE Journal of Solid-State Circuits, Vol. 32, No. 6. pp.861-869, June 1997.

[5] Z. Liu and V. Kursun, "New MTCMOS Flip-Flops with Simple Control Circuitry and Low Leakage Data Retention Capability," Proceedings of the IEEE International Conference on Electronics, Circuits, and Systems, pp. 1276-1279, December 2007.

[6] J. Kao, A. Chandrakasan, and D. Antoniadis, "Transistor Sizing Issues and Tool for Multi-Threshold CMOS Technology," Proceedings of the IEEE/ACM International Design Automation Conference, pp. 409 - 414, June 1997.

[7] B. H. Calhoun, F. A. Honore, and A. P. Chandrakasan, "A Leakage Reduction Methodology for Distributed MTCMOS," IEEE Journal of Solid-State Circuits, Vol. 39, No. 2, pp. 818 - 826, May 2004.

[8] S. A. Tawfik and V. Kursun, "Low-Power and Compact Sequential Circuits with Independent-Gate Fin FETs," IEEE Transactions on Electron Devices,

Vol. 55, Number 1, pp. 60-70, January 2008.

[9] R. Kumar and G. Hinton, “A Family of 45nm IA Processors,” Proceedings of the IEEE International Solid-State Circuits Conference, pp. 58-59, February2009.

[10] Z. Liu and V. Kursun, "High Read Stability and Low Leakage SRAM Cell Based on Data/Bitline Decoupling," Proceedings of the IEEE International Systems on Chip (SOC) Conference, pp. 115-116, September 2006.

[11] R. S. Muller, T. I. Kamins, and M. Chan, Device Electronics for Integrated Circuits, John Wiley \&Sons Ltd., 2002, ISBN \# 0471593982.

[12] http://www.umc.com/english/process/g.asp. UMC 90 Nanometer CMOS Technology.

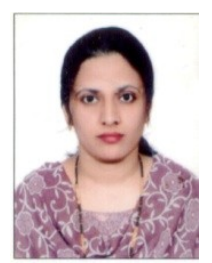

Tarunnum Sultana ${ }^{1}$ Assistant professor in Electronic and Communication engineering in SSJ College of Engineering. She received her B.Tech Degree in Electronics \& Communication Engineering from Gurunanak Dev Engineering College from Bidar, Karnataka and M.Tech Degree in DSCE from Shadan women's Engineering College Hyderabad and her current interest in Digital Electronics. 


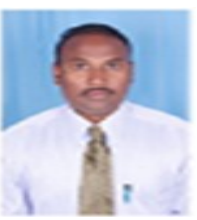

S. Jagadeesh ${ }^{2}$, Associate Professor and HOD in SSJ Engineering College, Hyderabad. He received his B. Tech Degree in Electronics \& Communication Engineering from SKDU, Ananthapur and $M$. Tech Degree in Electronics and Communication Engineering from JNTUH, Hyderabad. He was a Research scholar in Electronics \& communication Engineering Department, JNTUH. He has more than 13 years of experience in teaching and research. His current interest includes Digital image processing and electronics.

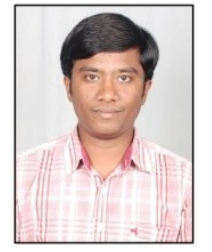

M. Naveen Kumar ${ }^{3}$, M.Tech-VLSI Student of SSJ Engineering College, JNTUH, Hyderabad. He is completed his B. Tech in Electronics and Communication Engineering from DRK Engineering college, JNTUH, Hyderabad. 